\title{
Cognitive Radio Ad Hoc Networks for Smart Grid Communications: A Disaster Management Approach
}

\author{
Hossein Khayami, Mohsen Ghassemi, Kamyar Ardekani, Behrouz Maham \\ School of ECE, College of Engineering \\ University of Tehran \\ Tehran, Iran \\ Email: \{h.khayami, moh.ghasemi, kamyar.ardekany, bmaham\}@ut.ac.ir
}

\author{
Walid Saad \\ ECE Department \\ University of Miami \\ Miami, USA \\ Email: walid@miami.edu
}

\begin{abstract}
The explosive growth in users' demand in both areas of wireless communications and power generation has led to design of new key technologies that will be dominant in the near future; cognitive radio networks in communications and smart grid in power field. This paper proposes a novel scenario to marry these technologies together by using a cognitive radio ad hoc network (CRAHN) as the foundation of smart grid communications. In particular, the formation and throughput of a citywide network, the information transferred by the network, and how this structure can be relied upon in disasters is discussed and compared to the state-of-the-art.

Index Terms-disaster management, cognitive radio, ad hoc networks, smart grid communications, femtocell.
\end{abstract}

\section{INTRODUCTION}

The smart grid (SG) is envisioned to bring about fundamental changes in electrical power distribution and generation networks in order to obviate shortages and limits of conventional grids $[1,2]$. Some of the most significant features which are made possible by realization of this smart grid vision are:

- Distributed generation of electrical energy;

- More dependability on renewable energy sources;

- Enhancement of customer service quality;

- Realization of new business models of demand response energy market mechanisms.

Due to its large-scale and distributed nature, two-way flow of information is an inevitable component of the smart grid infrastructure. Naturally, the transfer of critical grid information heavily relies on a capable communication infrastructure. In turn, this has resulted in significant research in the field of smart grid communications [3]. In such works, the main goal is to find, analyze and evaluate various communication technologies as potential solutions that can satisfy the requirements of smart grid communication infrastructure. Generally, there are two mainstream solutions, wired and wireless communications. The implementation of each of these technologies

This work was supported by the project numbered 91000529 entitled "Design and Performance Analysis of Smart Wireless Networks for Machineto-Machine Communications", funded by Iran National Science Foundation (INSF) and, in part, by the U.S. National Science Foundation under Grant CNS-1253731. in different parts of the smart grid network has its own advantages and drawbacks. In general:

Wired structures:

- Provide exclusive mediums for separate links, resulting in higher bandwidth and throughput.

- Are more dependable on infrastructure and prone to physical damages.

Wireless structures:

- Share the same medium with other wireless transmitters, leading to limited bandwidth and throughput.

- Are less dependable on infrastructure and more resistant to external damages [4].

One of the conditions in which wireless solutions outdo their wired alternatives, is during disasters; where using wireless technologies would make it easier to keep the communication infrastructure of the grid in service.

Nonetheless, a fundamental limitation for using wireless communication based on conventional technologies and regulations is the lack of sufficiently available frequency spectrum bandwidth.

The scarcity of the wireless radio spectrum is currently a prominent issue in the wireless communications industry. A large portion of wireless frequency spectrum is already licensed in many countries, leaving only a small share for newly emerging applications. However, studies such as [5] show that in average only a small percentage of the spectrum is utilized at a specific time in a specific location; indicating that the current wireless spectrum shortage is due to conventional wireless communications regulation schemes. To address this problem, the concept of cognitive radio has been recently proposed as a means for allowing an efficient co-existence between unlicensed, secondary users (SUs) and licensed, primary users (PUs) [6]. In the cognitive radio paradigm, the secondary users may use the licensed bands as long as primary users do not use them. As soon as a primary user begins transmitting in their band, the secondary users must vacate these frequencies and find other unused frequency bands. Using various functions, such as dynamic spectrum sensing 
and access, cognitive radio enables radios to monitor their surrounding radio environment so as to enable an opportunistic usage of spectrum by allowing the SUs to intelligently decide on which frequency bands (or spectrum holes) to use and at which time [7]. Ultimately, cognitive radio has shown to yield a more efficient utilization of the wireless spectrum, subsequently providing higher wireless capacities.

In addition, the information network of the grid may be used to transfer data that is helpful for disaster management authorities. Especially, information at early hours after occurrence of a disaster is extremely valuable [8].They may use this information to locate damages and plan for rescuing possible victims respectively.

The main contribution of this paper is to study the type of information that can be helpful to speed up rescuing procedure during and after a disaster and to propose a communication network which can reliably transfer this information. Furthermore, in normal situations when there are no disasters, the proposed infrastructure can serve as a solution for smart grid communications.

Generally, during a disaster, the communication infrastructure may get damaged and cannot be adequately relied on. To address this problem, we propose a novel architecture based on a cognitive radio ad hoc network (CRAHN). By efficiently exploiting cognitive abilities, the proposed network is capable of using higher capacities when more frequency spectrum is available, and is less prone to fluctuations in frequency availabilities and adverse SNR conditions. The wireless and ad hoc nature of this proposed network makes it less dependable on centralized communication infrastructure, therefore less vulnerable to local damages. Even if some parts of the network go out of service, the whole network would remain operational.

Our initial analysis shows that, within a large-scale, citywide deployment, the proposed architecture may suffer from increased delay and/or reduced throughput. To overcome this problem, we propose the use of base stations that can collect information from their associated cluster. Based on information-theoretic analyses of large CRAHNs, known as scaling laws, we will show the effect of adding base stations to network parameters.

The rest of this paper is organized as follows: Section II discusses the main issues pertaining to the management of disaster conditions, with a focus on communication resources defects. Section III describes the system model of the proposed CRAHN. It also briefly discusses femtocells as a potential application which can be deployed based on this structure. Finally, conclusions are drawn in Section IV.

\section{Disaster Management}

In the early hours after the occurrence of a large-scale disaster, such as earthquake, flood, and hurricane, three major steps have to be taken simultaneously to alleviate the disaster effects $[9,10]$.

- Regular information collection;
- Decision making regarding effective allocation of rescue resources;

- Providing high priority victims with rescue resources.

To manage a disaster situation, the responsible authorities are required to make quick and adequate decisions. Often, such a decision is made based on the information gathered at disaster management bases or control centers. Part of this information is extracted from direct observations of people or authorized forces, and the other part can be extracted from the SCADA (Supervisory Control And Data Acquisition) system of the smart grid or other sensory information collected at each node, i.e., each building.

Despite the vital importance of early information collection in disaster situations, it typically takes a long time for the first direct observations to be reported. Hence, automatic collection and transmission of sensory data can be extremely beneficial to disaster management and rescue resource allocation.

\section{A. Communication Requirements}

In order to remain operational in a catastrophic condition, the network which is used in emergency and disaster requires a number of specific functionalities and characteristics. Such as:

- Resistance to local damage: the communication network should not allow a local damage to spread to other sections which can eventually be detrimental to the whole system. Moreover, if some of the nodes lose their connectivity, other nodes must remain connected to the designated destination, i.e., one of the disaster management bases so as to transfer their collected information [9].

- Independence from users: the network should automatically send emergency information and must not require any manual operations by the user since they may not be in a normal situation.

- Low power consumption: the nodes must not consume high power and have to be able to operate without external power sources until the power grid is brought back in service or the situation back to normal operating conditions (i.e. fully controlled by first responders or rescue forces [11]. This implies that long-range transmission is undesirable, especially in higher frequencies. In turn, this motivates use of frequency channels such as the VHF and UHF frequency bands that are typically used for TV transmissions.

- Resistance to spectrum defects:the network must work in unoccupied bands which are not subject to severe interference from other wireless devices. This encourages the use of flexible radios to find bands with desired wireless characteristics.

Furthermore, in addition to being operational under disaster circumstances, the network should satisfy the minimum delay and throughput requirements. These requirements are determined by the amount and importance of information needed for rescue operations or other decision making plans and can be adaptable with respect to network conditions. In emergency 
TABLE I

DATA GATHERED IN NORMAL SitUation

\begin{tabular}{|c|c|c|}
\hline Smart Meters & Sensors & Phasor Measurement Unit \\
\hline \hline $\begin{array}{c}\text { Utility meters with two-way } \\
\text { communication }\end{array}$ & $\begin{array}{c}\text { Monitoring the mechanical and electrical } \\
\text { components of the grid }\end{array}$ & $\begin{array}{c}\text { Phasor measurement in different locations } \\
\text { to manage the power quality }\end{array}$ \\
\hline
\end{tabular}

TABLE II

Data Gathered During Disasters

\begin{tabular}{|c|c|c|}
\hline Smart Meters & Physical Damage Detectors & Human Status Detectors \\
\hline \hline $\begin{array}{c}\text { Consumption pattern monitoring } \\
\text { and fault detection }\end{array}$ & $\begin{array}{c}\text { Accelerometers } \\
\text { (shake and motion detector application) }\end{array}$ & CO2, voice, motion detectors \\
\hline
\end{tabular}

situations in which the available frequency bands are scarce, the information must be filtered and only necessary data should be transmitted, as discussed next.

\section{B. Smart grid and rescue data}

Nodes of this network consist of buildings, sensory nodes of the smart grids SCADA, and other crucial facilities. In each of these nodes, data is collected from the smart meters or other sensors. This data is subsequently sent to the decision making centers at the disaster management bases. Table I shows general categories of the data gathered in buildings and some of the related sensors.

At the disaster management bases, given the gathered data, the responsible entities must determine whether each home is abandoned or not and if a certain house is still occupied, estimate the number of people inside so as to assess the population distribution before and after the disaster. This can be, for example, done by analyzing the power consumption pattern of each household in the last hours just before the disaster. This process is called decision making.

Moreover, decision making is about identifying occurrence of a fault and instantly informing disaster management centers so that they can take immediate actions. Such a prompt emergency communication is critical because statistics have shown that most deaths happen in the first hours after a disaster [12].

1) Local vs. Centralized: Data processing can be done either locally at the level of each node before being transmitted to a disaster management center, or in a centralized fashion at a central monitoring center. There are benefits and drawbacks for each method. In centralized data collection, raw data must be sent to a monitoring center, such as the disaster management bases, on a regular basis and even more importantly, immediately after occurrence of an anomaly which can be an indication of a disaster. Naturally, transmitting such a large volume of data can increase the overall power consumption of the transmission system and it also requires a high throughput which cannot be guaranteed during a disaster since many nodes may start transmitting data simultaneously. Nonetheless, a central collection of data, generally, allows decision making bodies to make a more precise decision based on complete global data extracted from all nodes.

Local data processing involves filtering data and making basic decisions at each node. This can greatly reduce the amount of data that must circulate in the network as well as the power consumption of transmitters. However, it may lead to a decrease in the accuracy of decisions due to the availability of only a limited set of data (i.e., possible loss or lack of information).

Ideally, developing a system that can adaptively balance the tradeoff between these two scenarios is desirable. In other words, depending on the wireless spectrum availability, disaster characteristics, and disaster management approaches, the authorities can specify the level of localization. At one end of the spectrum, each node sends all its raw data received from its sensors, and at the other end, the nodes only send the results of the local decision making to the monitoring centers.

2) Prioritization: Each node of the ad hoc network receives data from different nodes that should also be sent along with its own data. Since important data must suffer less delay compared to less critical information, we propose a prioritization method in which each packet of data is assigned a priority index in the original node. This index is generated using a function:

$$
P_{i}=f\left(D_{i}, n_{i}, H_{i}, S_{i}\right)
$$

where $P_{i} \in[0,1]$ is the priority index of node $i, D_{i}$ is a measure of damage caused to the building $i$ which is calculated based on the sensory data, $n_{i}$ represents the number of residents inside the building at the time of disaster, $H_{i}$ is the degree to which the building is exposed to hazards such as nearby gas stations, and $S_{i}$ designates how strategic the site is. For instance, a hospital or a bank is of high strategic importance.

When congestion occurs at a node, it rejects forwarding data packets from each of its incoming ports with a probability proportional to the following:

$$
P\left\{\text { packetdrop }_{i}\right\}=1-P_{i}
$$

In this way, on the average, more bandwidth is provided for nodes with higher priority and they suffer less delay. At the same time, other nodes are allowed to transmit with more delay and less average throughput. 


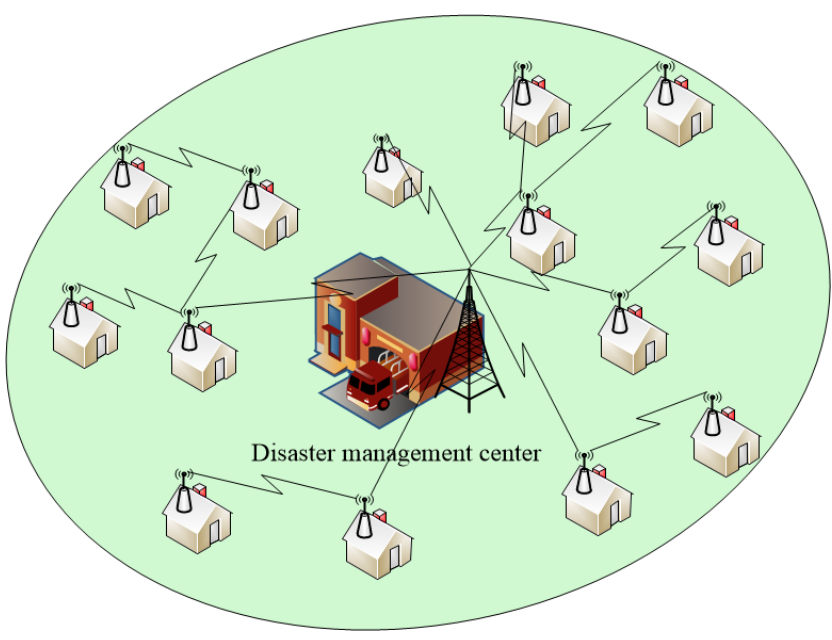

Fig. 1. Proposed CRAHN Topology

\section{Proposed Network Model}

\section{A. System Model}

As a solution to the communication requirements discussed in Section II, we propose a cognitive radio ad hoc network (CRAHN) as the communication infrastructure. The proposed architecture is ad hoc in the sense that no particular infrastructure is required to set up the network. Nodes connect to each other without prior configuration and deliver their information cooperatively using a multi-hop structure. As a result, localized damage due to a disaster would not lead to malfunction of the whole system. In case some of the nodes get damaged, the system can still connect functional nodes and pass information to the destination, namely, the nearest disaster management base. Figure 1 illustrates a view of this network, prior to any damage.

The system utilizes cognitive radio technology so that within each region, the nodes can opportunistically transmit in the frequency bands in which they detect no presence of primary users. As soon as a primary user is detected, the nodes are able to change their operating frequency band and use other unused bands as secondary users via well-known dynamic access schemes (see [13]).

In order to cooperatively pass information and reliably detect available frequency bands in the network, the nodes are grouped in clusters. Each cluster is composed of nodes that have similar decision about which frequency bands are available and which are not (such clustering is popular in cognitive radio networks, see [14]). The nodes in each cluster carry out distributed sensing and share their sensing information so as to decrease the probability of false detection. Between different clusters gateway nodes are deployed. These nodes serve as routing bridges between two clusters and must be able to communicate in a frequency band that is not occupied by primary users in either of the clusters. Figure 2 shows how nodes are laid out in clusters and their gateways.

There have been several efforts to analyze the capacity of such networks. Due to the flexible nature of node layouts

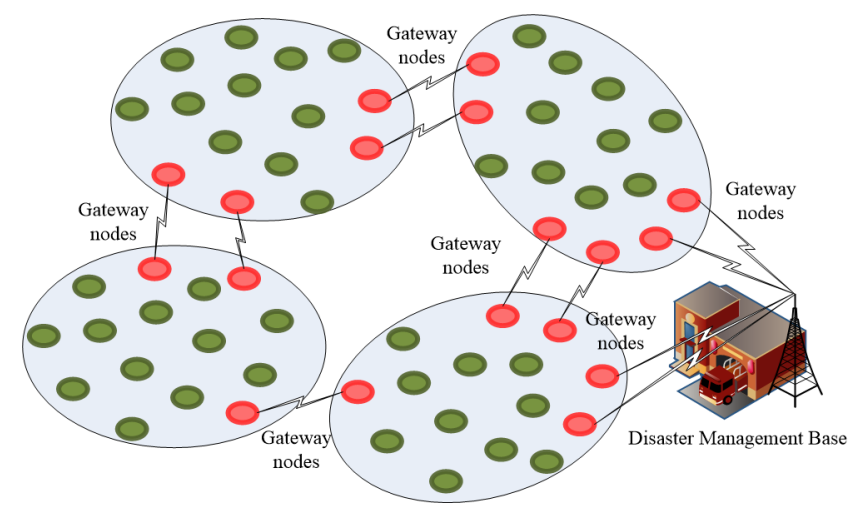

Fig. 2. Clusters and Gateways

and primary spectrum usage, commonly, upper bounds and lower bounds of the capacity are of interest. Also, since in networks of interest, the number of nodes is very large, usually asymptotic analysis of network capacity is provided (see [15, $16,17])$. In our proposed network, this assumption is valid, because the number of buildings, which of each corresponding to one node in the network, is large.

As shown in the next subsection, the throughput of each node declines as the number of nodes increases. As a result, the whole system throughout within a large-scale deployment such as in a big city cannot be a purely ad hoc network. Base stations should be deployed throughout the network. Consistently, they can be installed in disaster management bases, which are supposed to be reliable and resistant to damages caused by large-scale disasters. These stations are connected to each other and to the central management base using high-speed wired or a satellite backhaul. Figure 3 shows how such base stations can be laid out and connected to one other.

\section{B. Scaling Laws}

Extensive research has been done to derive scaling laws of various cognitive ad hoc networks based on different assumptions and scenarios. Here, we bring some of the findings to demonstrate how per-node throughput asymptotically scales with number of nodes.

In [18], it is shown that under certain assumptions, which are valid in our proposed network model, both secondary and primary networks achieve the same throughput scaling as when the other network is absent. These assumptions include:

- Secondary nodes are aware of the locations of primary nodes, however, primary nodes need not be aware of existence of the secondary network;

- Secondary nodes are denser than primary nodes.

It is shown in [16] that in a random general CRAHN:

$$
\left.\left.\lambda_{n} \in\left[\Omega\left(\frac{C_{\alpha}}{\sqrt{n \ln n}}\right)\right), O\left(\frac{C_{\zeta}}{{ }_{n} 1 / \lambda}\right)\right)\right]
$$

where $\lambda(n)$ is the per-node throughput for a network with $n$ nodes, $C_{\alpha}$ and $C_{\zeta}$ are respectively the minimum link capacity 


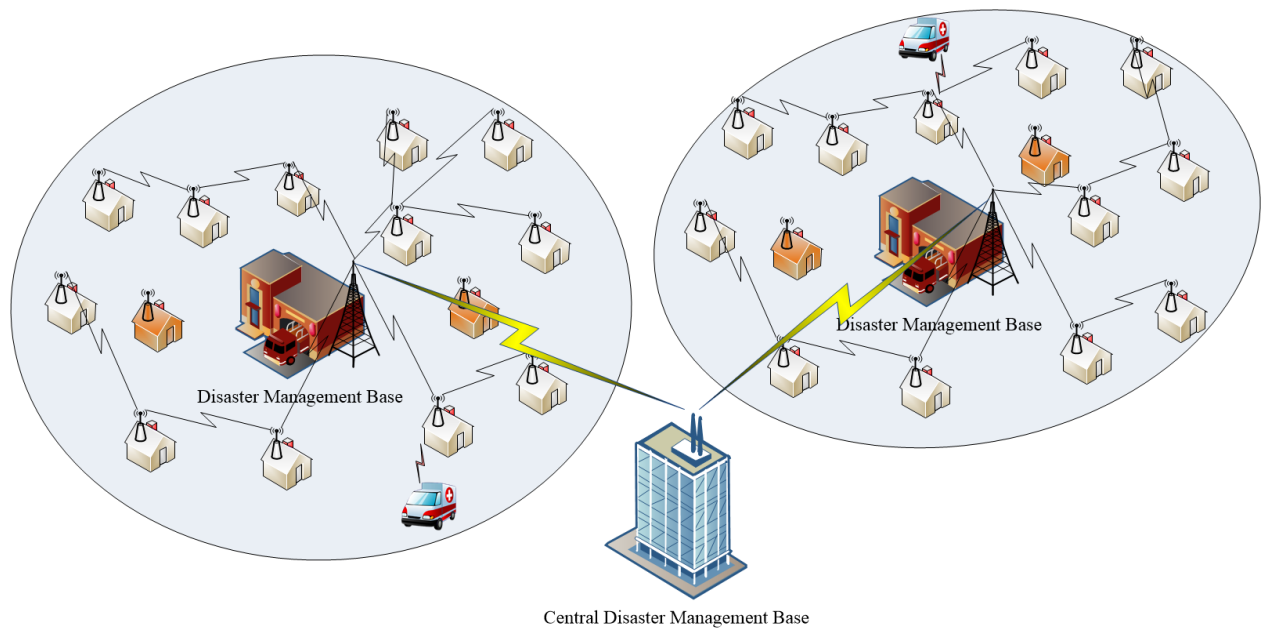

Fig. 3. CRAHN with Nodes Assigned to Various Base Stations

over all links and sum of capacity over all available bands in the CRAHN, and $\gamma \geq 2$ is the path loss index.

The expression in [3] gives an insight on how the increase of the node numbers results in a decrease of the per-node throughput and encourages the idea of using disaster management bases as base stations. Thus, by limiting the number of nodes assigned to each station we are able to provide the minimum required throughput for each node.

Furthermore, it is shown in [19] that with adoption of hierarchical cooperation methods between nodes of the network, i.e., use of clustering and MIMO techniques, achieving the optimal capacity (that is $\left.\mathrm{O}\left(\frac{C_{\zeta}}{n 1 / \lambda}\right)\right)$ is possible.

\section{CRAHN Nodes as Femtocells}

The concept of small-cell networks has been used for increasing capacity of cellular networks. Femtocells or home base stations are the most recent members of this family which have lately attracted a lot of [20]. Assuming the conventional cellular networks are out of service as a result of the disaster, the frequency bands assigned to them are considered white spaces. Thus, considering significant frequency reuse of femtocell networks (FNs), these bands can be used for communication between mobile users and home base-stations. In contrast with the conventional FNs which have wired backhaul, our proposed FN uses the introduced wireless CRAHN to transmit data between buildings. Switching to FN coverage can be triggered immediately after identifying a disaster and terminated when the cellular network is restored to its normal condition. The objective of the FN is merely to enable making emergency voice calls or sending voice/text messages for help request to rescue centers and contacts among users or other regular services are not allowed inasmuch as communication networks are prone to congestion in critical situations.

\section{CONCLUSION}

In this paper, we have discussed how the use of CRAHNs as one an infrastructure of smart grid communications can simultaneously benefit dependable data transfer in smart grid and help disaster management teams to make timely decisions based on smart grid data and other sensory information from the building. Furthermore, we have analyzed some of the requirements which the network structure should satisfy during a disaster. Based on these requirements, a CRAHN network with interoperability features was proposed and described.

Then, we have discussed how this network can also serve as a femtocell network to provide cellular communication service during a disaster.

This paper provided the general view of the idea of using CARHN as a communication infrastructure for both smart grid and disaster management. For future work, a precise, closedform expression for the relation of throughput and delay of the network as a function of parameters such as the number of base stations, the layout of gateways, and the probability of false empty band detection, can be analyzed.

\section{REFERENCES}

[1] X. Fang, S. Misra, G. Xue, and D. Yang, Smart Grid - The New and Improved Power Grid: A Survey, IEEE Communications Surveys and Tutorials, in press.

[2] A. Kwasinski, "Towards a Power-Net: Impact of smart grids development for ICT networks during critical events," 3rd International Symposium on Applied Sciences in Biomedical and Communication Technologies (ISABEL), pp.1-8, November 2010.

[3] Alizadeh, M.; Xiao Li; Zhifang Wang; Scaglione, A.; Melton, R., "Demand-Side Management in the Smart Grid: Information Processing for the Power Switch," Signal Processing Magazine, IEEE , vol.29, no.5, pp.55,67, Sept. 2012

[4] A. P. Athreya and P. Tague, "Survivable smart grid communication: Smart-meters meshes to the rescue," International Conference on Computing, Networking and Communications (ICNC), pp.104-110, January 2012.

[5] Cognitive Radio Technologies Proceeding, Federal Communications Commission, Rep. ET Docket No. 03-108, http://www.fcc.gov/oet/cognitiveradio/.

[6] Haykin, Simon, "Cognitive radio: brain-empowered wireless communications," Selected Areas in Communications, IEEE Journal on , vol.23, no.2, pp.201,220, Feb. 2005.

[7] I. F. Akyildiz, W. Lee, and K. R. Chowbury, CRAHNs: Cognitive radio ad hoc networks, Ad Hoc Networks Journal, vol. 7, pp. 810-836, July 2009. 
[8] K. Ishizu, H. Murakami, and H. Harada, Cognitive wireless network infrastructure and restoration activities for the earthquake disaster, 14th International Symposium on Wireless Personal Multimedia Communications (WPMC), pp. 1-5, October 2011.

[9] Y. N. Lien, T. C. Tsai, and H. C. Jang., "A MANET Based Emergency Communication and Information System for Catastrophic Natural Disasters," 29th IEEE International Conference on Distributed Computing Systems Workshops, pp.412-417, June 2009.

[10] N. Uchida, K. Takahata, and Y. Shibata, "Cognitive Wireless Network for Large Scale Disaster," 3rd International Conference on Intelligent Networking and Collaborative Systems (INCoS), pp.362-366, November 2011.

[11] Y. Hiehata, H. Koto, and H. Nakamura, "A Proposal of a Communication-Broadcasting Integrated System to Support Communication and Navigation during Disasters," 5th International Conference on Internet Monitoring and Protection (ICIMP), pp.110-116, May 2010.

[12] S. Majid and K. Ahmed, Post-Disaster Communications: A Cognitive Agent Approach, 7th International Conference on Networking, pp. 645650, April 2008.

[13] E. Hossain, D. Niyato, and Z. Han, Dynamic Spectrum Access and Management in Cognitive Radio Networks, NY: Cambridge University Press, 2009

[14] W. Saad, Z. Han, M. Debbah, A. Hjrungnes and T. Baar, "Coalitional Games for Distributed Collaborative Spectrum Sensing in Cognitive Radio Networks," in Proc. of the IEEE International Conference on Computer Communications (INFOCOM), Rio de Janeiro, Brazil, April 2009.

[15] M. Vu, N. Devroye, and V. Tarokh, "An overview of scaling laws in ad hoc and cognitive radio networks," Wireless Personal Communications, vol. 45, no. 3, pp. 343-354, May 2008.

[16] Y. Shi, C. Jiang, Y. Thomas Hou, and S. Kompella, On capacity scaling law of cognitive radio ad hoc networks, Proceedings of 20th International Conference on Computer Communication Networks (ICCCN), pp.1-8, July 2011.

[17] O Lvłque, and I.E. Telatar, Information-theoretic upper bounds on the capacity of large extended ad hoc wireless networks, IEEE Trans. Inf. Theory, vol. 51, No. 3, pp. 858865, 2005.

[18] S.W. Jeon, N. Devroye, M. Vu, S.Y. Chung, and V. Tarokh, Cognitive networks achieve throughput scaling of a homogenous network, IEEE Trans. On Information Theory, vol. 57, pp. 5103-5115, August 2011.

[19] A. zgr, O. Lvlque, and D. N. C. Tse, Hierarchical cooperation achieves optimal capacity scaling in ad hoc networks, IEEE Trans. Inf. Theory, vol. 53, pp. 3549-3572, October 2007.

[20] V. Chandrasekhar, J. G. Andrews, and A. Gatherer, Femtocell Networks: A Survey, IEEE Communications Magazine, Vol. 46, No. 9, pp. 59-67, September 2008. 